\title{
A Double End Fault Location Technique for Distribution Systems Based on Fault-Generated Transients
}

\author{
F. M. Aboshady*†, Mark Sumner*, D. W. P. Thomas* \\ * Department of Electrical and Electronic Engineering, University of Nottingham, Nottingham, UK \\ $\dagger$ Electrical Power and Machines Engineering Department, Tanta University, Tanta, Egypt \\ fathy.aboshady@nottingham.ac.uk
}

\begin{abstract}
This paper presents a fault location technique for distribution systems. It is a two end impedance based technique that uses the fault generated transients to estimate the fault distance over a broad range of frequencies. Then, curve fitting is applied to find the final estimated fault distance. Firstly, the paper introduces the method for the system represented as a lumped RL model. Then, generalized to consider the distribution line capacitance. The technique accounts for presence of loading taps, heterogeneous feeder sections, single phase, two phase and three phase loads and unbalance in distribution system. Single line to ground, line to line, and three phase faults are considered at different fault resistance values up to $100 \Omega$. Also, the effect of fault inception angle and resolution of analogue to digital converter is investigated. IEEE 34 nodes system is used to evaluate the proposed method.
\end{abstract}

Keywords-fault location; fault transients; impedance based techniques.

\section{INTRODUCTION}

Fast and accurate fault location can improve power system continuity by reducing the time required to locate and repair a permanent fault and therefore reduce the interruption time. It can also help with locating intermittent or high impedance faults, providing information to maintenance teams of the weak points in a system [1]. It is becoming an increasingly important topic as distribution systems become more flexible and complicated for example with the increase of embedded generation.

Several techniques for fault location have been developed based on a variety of methods including apparent impedance, travelling wave, artificial intelligence and disbursed devices based techniques [2]. The impedance based methods mainly use the fundamental frequency components to estimate the fault distance: Furthermore they can be classified to single end and multi terminal techniques based on the availability of measurement points in the system [3-6]. The single end methods are cheap and simple and use iterative approaches to compensate for the unknown fault resistance value and remote end current which in turn affect the method accuracy. On the other hand, multi terminal methods depend on several measurements which increase both accuracy and cost. Impedance based techniques that use the fault generated high frequencies were reported in $[7,8]$. These wideband techniques have the advantage of using a short data window, hence they could be implemented in real time applications with a fast response time. Travelling wave techniques detect the high frequency waves reflected from the fault point and use the arrival time for these waves to the measurement point(s) to estimate the fault distance [9]. Despite of being fast and accurate, they require very high sampling rates in range of $\mathrm{MHz}$ or even GHz. Also, for application with distribution systems, the waves will suffer from more reflections and attenuation due to presence of loading taps and lateral connections. Therefore, the implementation of travelling wave based techniques in distribution systems is complex and cost intensive. Artificial intelligence based techniques employ a heuristic way of using the data collected from the system. Using artificial neural networks is an example for such techniques [10]. These methods require extensive training (and retraining if the system topology changes) to accommodate for all possible fault scenarios. Techniques that can benefit from devices installed along the system such as smart meters or fault indicators have been reported [11, 12]. Usually these methods define the closest bus to the fault point instead of the actual fault position. In [11], a method based on matching the recorded voltage sag and the calculated voltage sag simulated at all system nodes was presented: the system node with the highest match is considered to be the faulty or closest node to the fault point. The benefit behind the method is the ability to locate subcycle faults. However, it assumed the availability of measurements at all system nodes and will fail if this is not available.

Over recent years, distribution system have become more instrumented with an increase in the number of deployed measurement devices [13]. Fault location techniques could be improved by using these devices. In this paper, a fault location algorithm is presented that considers the presence of measurement at both substation and the end node of the feeder. The proposed technique is classified as an impedance based technique. Similar to $[7,8]$, the proposed technique uses the high frequency transients 
generated from the fault to estimate the fault distance. However, in [7], the algorithm is based on the presence of a re-closer circuit breaker at the beginning of each section in the feeder which is impractical. Only solid faults were examined using this algorithm, hence the accuracy against fault resistance is not guaranteed. In [8], the algorithm assumed the availability of measurements at both ends of every section, and a sampling frequency of $1 \mathrm{MHz}$ was necessary which increases the cost of implementation. When the algorithm was applied to a system with loading taps between the two measurement points, the accuracy was significantly decreased and a percentage error of $25 \%$ in the estimated distance was obtained.

The proposed technique only requires a short data window - less than one cycle during and before the fault occurrence - and a sampling frequency of $20 \mathrm{kHz}$ to estimate the fault distance. The method is suitable for real time implementation on a simple microcontroller. Also, it is able to locate sub-cycle faults, helping to reduce the number of unexpected outages. The proposed technique considers the presence of loading taps, non-homogeneous feeder sections, system unbalance, different fault types, different fault resistance values, various fault inception angles and influences from the data acquisition equipment. Also, the derivation considers a distribution system without line capacitance and then is generalized to include the capacitance of the distribution lines.

\section{METHODOLOGY}

The fault location technique presented in this paper depends on the fault transients generated when a fault occurs. The technique is firstly derived ignoring the line capacitance, then the procedure is generalized to consider the line capacitance. The proposed method is carried out based on phase analysis rather than sequence components analysis to consider the unbalance that might exist in the distribution systems.

\section{A. RL Lumped Parameters System}

Assuming a fault at point $f$ between any two nodes $S$ and $R$, the fault is modelled as a source for a transient at the fault point as shown in Fig. 1. The voltage at point $f$ is obtained by (1) and (2).

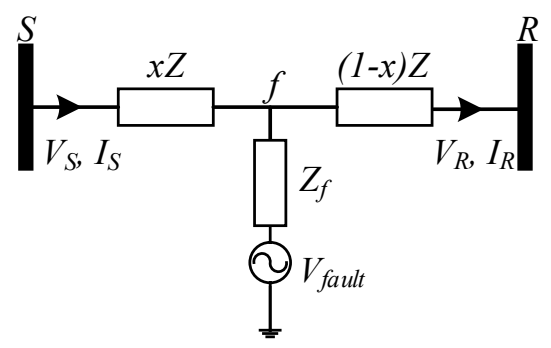

Fig. 1. Single line diagram for system with a fault at point $f$

$$
\begin{gathered}
V_{f}=V_{S}-x Z I_{S} \\
V_{f}=V_{R}+(1-x) Z I_{R}
\end{gathered}
$$

where $x$ is the per unit fault distance measured from node $S$; $V_{S}$ and $I_{S}$ are vectors of three phase voltages and currents at node $S ; V_{R}$ and $I_{R}$ are vectors of three phase voltages and currents at node $R ; Z_{f}$ is the fault impedance; $Z$ is the total section series impedance including both self and mutual impedances.

The voltage and current vectors at nodes $S$ and $R$, are obtained by wideband measurements at substation downstream and wideband measurements at the feeder end node upstream respectively [14]. By equating (1) and (2), the following equation as a function of the per unit fault distance $x$ is obtained:

$$
x Z\left(I_{S}-I_{R}\right)=V_{S}-V_{R}-Z I_{R}
$$

Equation (3) is used to calculate the three values for $x$, these values correspond to the three phases. By applying (3) at different frequencies generated from the fault, there will be a series of values for $x$ for each phase. Curve fitting is used to find the most appropriate value of $x$ for each phase. Curve fitting is applied on results within the frequency range $500 \mathrm{~Hz}$ to $2000 \mathrm{~Hz}$. The values that correspond to the faulty phases are considered. Also, for multi-phase faults, the average value for the values of $x$ is used as the fault distance, e.g. for a fault between phases $\mathrm{A}$ and $\mathrm{B}$, the final fault distance is the average of the two values for $x$ for phases $\mathrm{A}$ and $\mathrm{B}$.

\section{B. System with Capacitance}

In this part the technique considering the line capacitance is derived. The general configuration is shown in Fig. 2. The same procedure is applied but includes the line capacitance. Here, the line is modelled using the general ABCD parameters. Considering a $\pi$ model for the system, the values for $A_{1}, B_{1}, A_{2}$, and $B_{2}$ as a function of $x$ are given by (4)

$$
\begin{gathered}
A_{1}=I+0.5 x^{2} Z Y \\
B_{1}=x Z \\
A_{2}=I+0.5(1-x)^{2} Z Y \\
B_{2}=(1-x) Z
\end{gathered}
$$

where $I$ is the identity matrix and $Y$ is the total section shunt admittance.

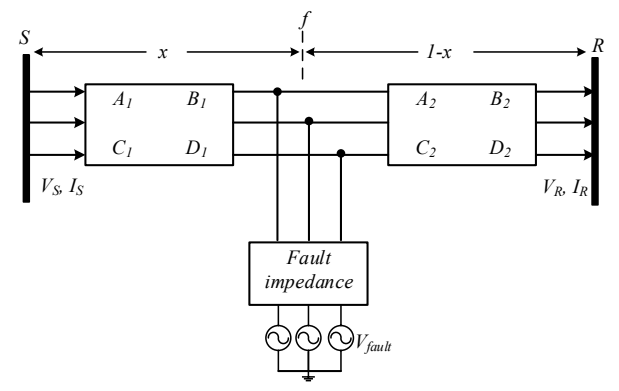

Fig. 2. Three phase system considering the line capacitance with a fault at point $f$ 
Also, the voltage at the fault point $f$ is given by (5) and (6)

$$
\begin{gathered}
V_{f}=A_{1} V_{S}-B_{1} I_{S} \\
V_{f}=A_{2} V_{R}+B_{2} I_{R}
\end{gathered}
$$

By equating (5) and (6) and substituting for the line parameters, a second order equation to find $\mathrm{x}$ is obtained.

$$
A A x^{2}+B B x+C C=0
$$

where,

$$
\begin{gathered}
A A=0.5 Z Y\left(V_{S}-V_{R}\right) \\
B B=Z\left(I_{R}-I_{S}\right)+Z Y V_{R} \\
C C=V_{S}-V_{R}-0.5 Z Y V_{R}-Z I_{R}
\end{gathered}
$$

By solving (7), the value of $x$ is calculated and the same procedure of curve fitting to find the final fault distance is followed.

As clear from the previous analysis, the fault distance estimation is independent of the fault resistance and the same equation is applicable for different fault types which allows the method to be simply implemented.

\section{CASE STUDY}

The IEEE 34 nodes system is used to evaluate the performance of the proposed method [15]. The test considers the longest feeder that extends from substation at node 800 to node 848 and the effect of laterals has not been covered in this study. The total length of this feeder is about $58 \mathrm{~km}$. This feeder is characterized by non-homogeneous sections and also the presence of single phase, two phase, and three phase (balanced and unbalanced) loads along the feeder. The simulation is carried out using Matlab/Simulink software with all loads treated as a constant impedance. Three phase voltage and current measurements at nodes 800 and 848 are used by the fault locator. A data window of 30 $\mathrm{ms}$, composed of pre-fault and during-fault segments is enough for the proposed method to estimate the fault distance. A sampling frequency of $20 \mathrm{kHz}$ is used for capturing the signals. Single line to ground, line to line and three phase faults are examined. The effects of fault resistance, fault inception angle and the use of a 16 bit analogue to digital converter resolution on the accuracy of the fault location are studied.

\section{A. Results for RL Lumped Parameters System}

\section{1) Effect of fault resistance}

Different fault types are simulated along the feeder at fault resistance values of $0.01,5,40$ and $100 \Omega$. The fault cases are carried out at fault inception angle of $30^{\circ}$. Fig. 4 presents the error in meters as calculated by (9) for different types of fault.

$$
\text { error }=\text { estimated distance }- \text { actual distance }
$$

For different fault resistance values, the error in estimated distance has a very close behavior. This reflects the independency of the proposed method on the fault resistance.

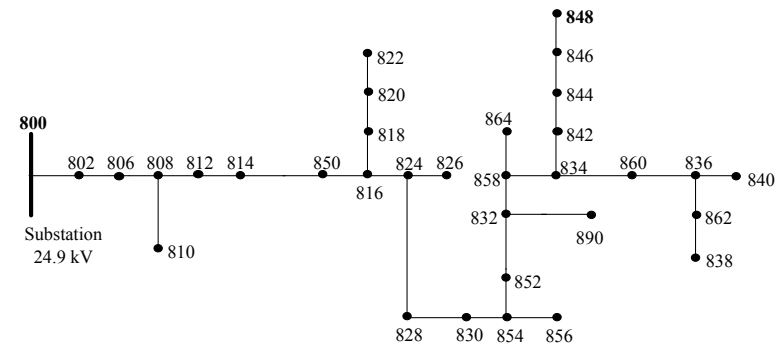

Fig. 3. Single line diagram for IEEE 34 nodes system

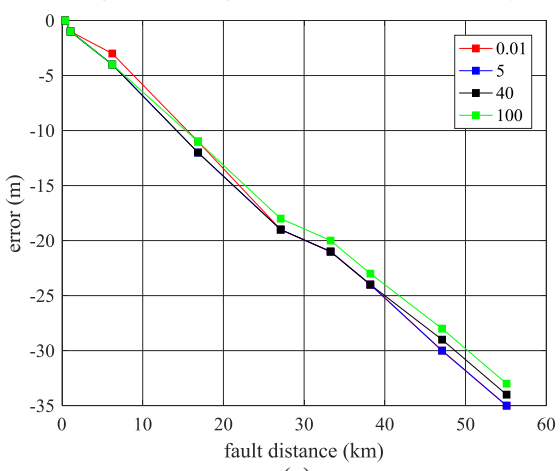

(a)

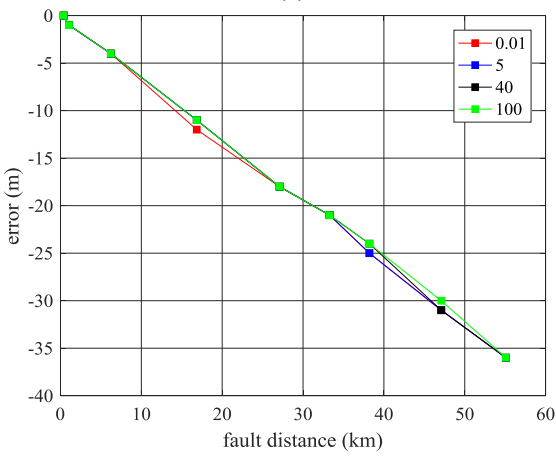

(b)

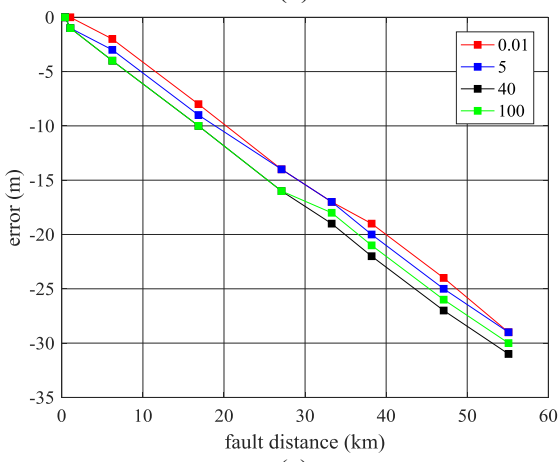

(c)

Fig. 4. Error in estimated distance at different fault resistance values, RL model, for (a) single line to ground fault, (b) lint to line fault and (c) three phase fault

\section{2) Effect of fault inception angle}

Five different values for the fault inception angle are used $\left(0^{\circ}, 30^{\circ}, 90^{\circ}, 120^{\circ}\right.$ and $\left.180^{\circ}\right)$, to evaluate of its influence on the proposed method. The result for a SLG faults with a fault resistance of $0.01 \Omega$ is illustrated in Fig. 5. Both $0^{\circ}$ and $180^{\circ}$ angles provide the same response as is clear from the figure legend. 


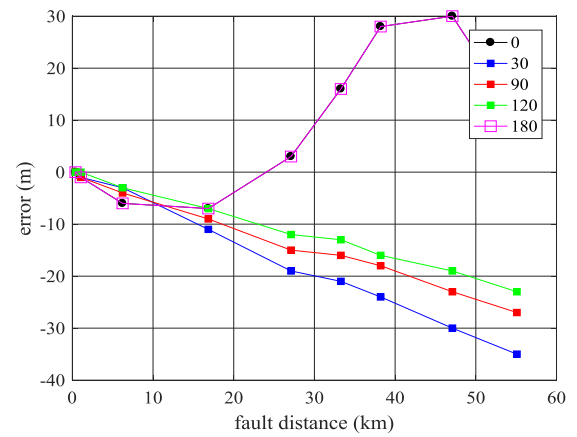

Fig. 5. Error in estimated distance for single line to ground fault at different fault inception angles, RL model

It can be seen from Fig.5 that a good estimate of fault location in this system can be achieved even when the phase voltage at the time of the fault is low (ie inception angle of $0^{\circ}$ or $180^{\circ}$ ).

From the previous results, it becomes clear that the proposed method is robust against fault distance, fault type, fault resistance value and the fault inception angle. The maximum absolute error obtained is about $35 \mathrm{~m}$ for a $58 \mathrm{~km}$ long feeder.

\section{B. Results for System with Capacitance}

For these results the model of the line used in the simulation has been modified to incorporate capacitance using the $\pi$ model, and the fault location method has been modified by using the general ABCD parameters for lines.

For a phase A to ground fault in the section between nodes 854 and 852, Fig. 6 illustrates the captured voltage and current at both measurement points for phase A. The negative time represents the pre-fault period. The oscillations that appear in the voltage signals are due to the effect of the system capacitance.

\section{1) Effect of fault resistance}

The three fault types investigated in this paper are carried out at seven points along the feeder at different fault values. All cases consider a fault inception angle of $30^{\circ}$.
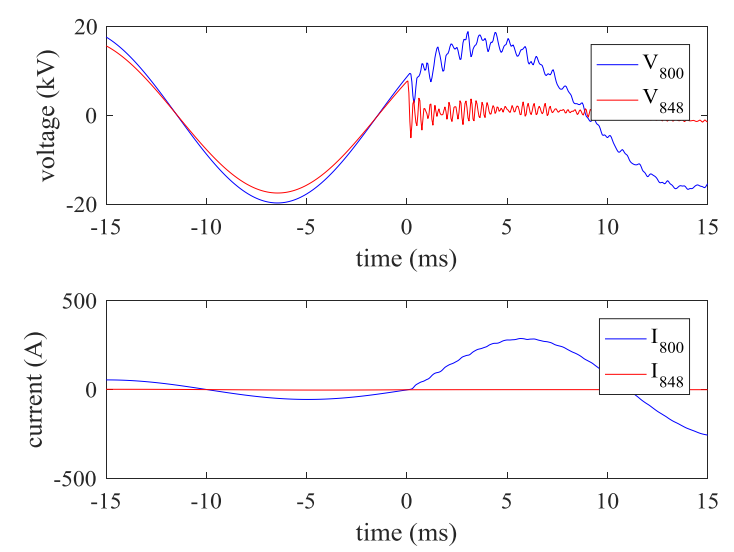

Fig. 6. Measured voltage and current for phase A to ground fault

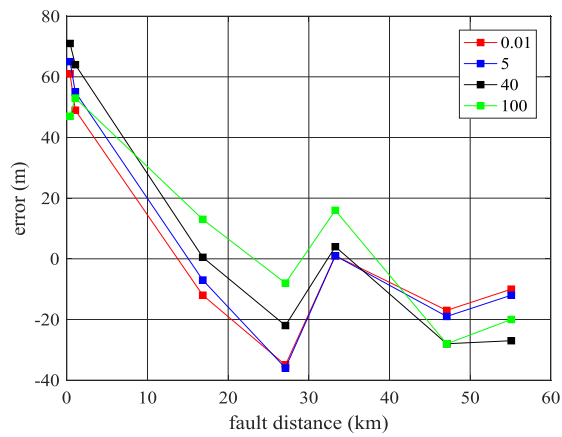

(a)

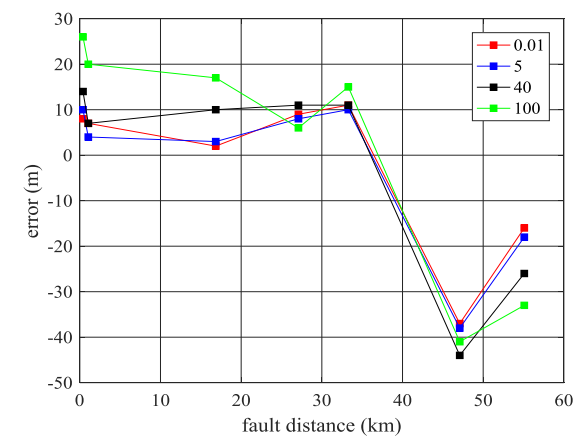

(b)

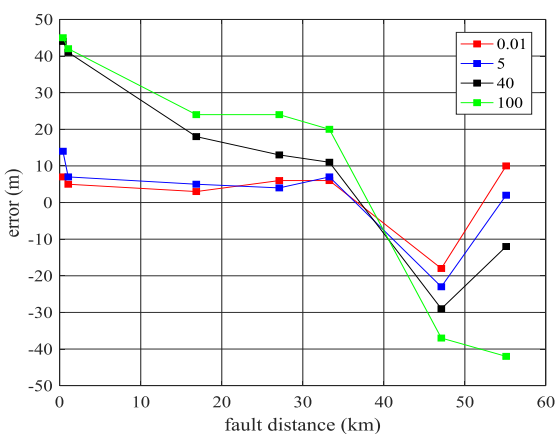

(c)

Fig. 7. Error in estimated distance at different fault resistance values, $\pi$ model, for (a) single line to ground fault, (b) lint to line fault and (c) three phase fault

\section{2) Effect of fault inception angle}

Fig. 8 illustrates the results for three phase faults with a fault resistance value of $5 \Omega$ simulated at five different fault inception angles.

From Fig. 7 and Fig. 8, although the accuracy of the method decreased compared to RL model, the method still works with a high accuracy that does not exceed $80 \mathrm{~m}$.

\section{3) Effect of 16bit ADC resolution}

A 16bit quantiser is applied to the measurements made in the simulation to consider the effect of the resolution of real equipment - in this case the analogue to digital converter - on the accuracy of the method. SLG, line to line and three phase faults are simulated at a fault resistance of 5 $\Omega$ and a fault inception angle of $30^{\circ}$. The error in estimated distance obtained by (9) is presented in Fig. 9. 


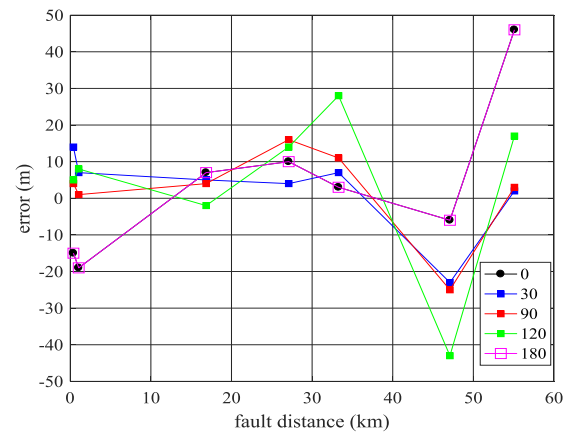

Fig. 8. Error in estimated distance for three phase fault at different fault inception angles, $\pi$ model

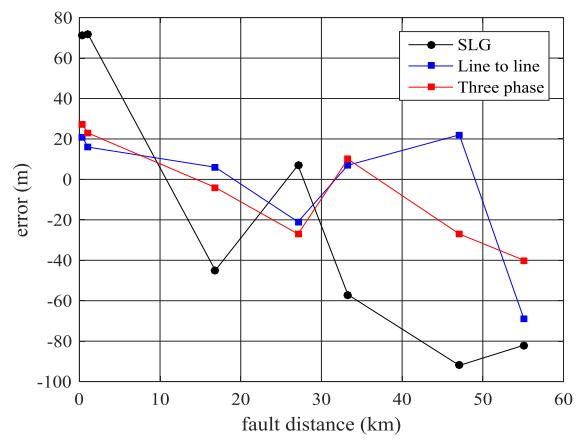

Fig. 9. Error in estimated distance when applying analogue to digital convereter, $\pi$ model

From all the previous results, the maximum absolute error obtained by the proposed method is less than $100 \mathrm{~m}$ for a total feeder length of about $58 \mathrm{~km}$.

\section{CONCLUSION}

A fault location technique based on the generated fault transients and using two end measurements has been presented. The technique considered several factors that affect accuracy of the fault location e.g. fault type, fault resistance, fault inception angle and resolution of the data acquisition equipment as well as some characteristics of the distribution system. The technique has the advantage of being fast and accurately pinpointing the fault location which suggests it can be used for real time applications and locating sub-cycle faults. Also, the proposed technique is independent of the fault resistance and the same equation is applicable to different fault types. Under all test conditions provided in this paper, the absolute error obtained was always less than $100 \mathrm{~m}$ for a total feeder length of about 58 $\mathrm{km}$. On-going work is investigating how the algorithm can compensate for the presence lateral connections and branches in the distribution system.

\section{ACKNOWLEDGMENT}

This work was supported by the Egyptian Governmentministry of higher education (cultural affairs and missions sector) and the British Council through Newton-Mosharafa fund.

\section{REFERENCES}

[1] M. M. Saha, J. Izykowski, and E. Rosolowski, Fault location on power networks: Springer, 2010

[2] M. Abad, M. García-Gracia, N. E. Halabi, and D. L. Andía, "Network impulse response based-on fault location method for fault location in power distribution systems," IET Generation, Transmission \& Distribution, vol. 10, pp. 3962-3970, 2016.

[3] R. Krishnathevar and E. E. Ngu, "Generalized Impedance-Based Fault Location for Distribution Systems," IEEE Transactions on Power Delivery, vol. 27, pp. 449-451, 2012.

[4] F. M. Abo-Shady, M. A. Alaam, and A. M. Azmy, "Impedancebased fault location technique for distribution systems in presence of distributed generation," in 2013 IEEE International Conference on Smart Energy Grid Engineering (SEGE), 2013, pp. 1-6.

[5] J. Ramirez-Ramirez, J. Mora-Florez, and C. Grajales-Espinal, "Fault location method based on two end measurements at the power distribution system," in Circuits \& Systems (LASCAS), 2015 IEEE 6th Latin American Symposium on, 2015, pp. 1-4.

[6] J. J. Mora-Florez, R. A. Herrera-Orozco, and A. F. Bedoya-Cadena, "Fault location considering load uncertainty and distributed generation in power distribution systems," IET Generation, Transmission \& Distribution, vol. 9, pp. 287-295, 2015.

[7] K. Jia, Z. Ren, T. Bi, and B. Liu, "A fault location method based on reclosing in distribution systems," in $20155^{\text {th }}$ International Conference on Electric Utility Deregulation and Restructuring and Power Technologies (DRPT), 2015, pp. 1016-1019.

[8] K. Jia, D. W. P. Thomas, and M. Sumner, "A New double-ended fault-location scheme for utilization in integrated power systems," IEEE Transactions on Power Delivery, vol. 28, pp. 594-603, 2013.

[9] A. Dwivedi and X. Yu, "Fault location in radial distribution lines using travelling waves and network theory," in 2011 IEEE International Symposium on Industrial Electronics, 2011, pp. 10511056.

[10] C. Apisit, C. Positharn, and A. Ngaopitakkul, "Discrete wavelet transform and probabilistic neural network algorithm for fault location in underground cable," in 2012 International conference on Fuzzy Theory and Its Applications (iFUZZY2012), 2012, pp. 154157.

[11] P. C. Chen, V. Malbasa, and M. Kezunovic, "Locating sub-cycle faults in distribution network applying half-cycle DFT method," in T\&D Conference and Exposition, 2014 IEEE PES, 2014, pp. 1-5.

[12] F. Trindade, W. Freitas, and J. C. d. M. Vieira, "Fault location in distribution systems based on smart feeder meters," in PES General Meeting | Conference \& Exposition, 2014 IEEE, 2014, pp. 251-260.

[13] M. Kezunovic, "Smart Fault Location for Smart Grids," IEEE Transactions on Smart Grid, vol. 2, pp. 11-22, 2011.

[14] W. H. Kersting, Distribution system modeling and analysis, 2 ed.: Taylor \& Francis Group, Boca Raton, 2007.

[15] IEEE PES Distribution System Analysis Subcommittee, 34-bus feeder[Online].Available:

http://ewh.ieee.org/soc/pes/dsacom/testfeeders/index.html 